\title{
Effects of phosphorous doping on the superconducting properties of $\operatorname{SmFeAs}(\mathbf{O}, \mathrm{F})$
}

Shiv Jee Singh ${ }^{*}$, Jun-ichi Shimoyama, Akiyasu Yamamoto, Hiraku Ogino and Kohji Kishio

Department of Applied Chemistry, The University of Tokyo, 7-3-1 Hongo, Bunkyo-ku, Tokyo 113-8656 Japan

*Corresponding author.

E-mail address: shivjees@gmail.com; tsjsingh@mail.ecc.u-tokyo.ac.jp

Tel.:+81-3-5841-7766

Fax: +81-3-5689-0574. 


\begin{abstract}
The systematic doping effect induced by the isovalent substitution of $\mathrm{P}$ for As on the superconducting properties of F-doped $\mathrm{SmFeAsO}_{0.88} \mathrm{~F}_{0.12}(\mathrm{Sm} 1111)$ has been studied by physical and magnetic measurements. The cell volume $(V)$ decreases with $\mathrm{P}$ doping and the anisotropic chemical pressure might be induced. However, the superconducting transition temperature $\left(T_{\mathrm{c}}\right)$ and the upper critical field $\left(H_{\mathrm{c} 2}\right)$ are suppressed. Thermoelectric power $(S)$ indicates the majority of electron type charge carriers in support of Hall measurements and its magnitude does not change very much for different $\mathrm{P}$ concentrations. The present investigation depicts that isovalent substitutions in the FeAs layer strongly deteriorate the superconducting properties of Sm1111 as a result of increase in chemical pressure. These isovalent substitution effects are comparatively discussed with hole $(\mathrm{Mn})$ and electron (Ni) type substitutions in the superconducting layer of Sm1111.
\end{abstract}

Keywords: Oxypnictide superconductors, transition temperature, upper critical field 


\section{Introduction}

Carrier doping is generally a powerful technique to induce superconductivity in the newly discovered iron pnictides [1]. Either electrons or holes are supplied to the $\mathrm{Fe} 3 \mathrm{~d}$ bands by elemental substitution and defect formation that may suppress the magnetic ordering of parent compound and induce superconductivity [2]. The iron pnictide REFeAsO (RE1111; RE = rare earth) consists of alternate layers of FeAs (conducting layer) and REO layer (charge reservoir) [3], and systematically studied by various kinds of doping. The highest transition temperature $\left(T_{\mathrm{c}}\right)$ of RE1111 ( $\left.\sim 58 \mathrm{~K}\right)$ has been achieved by electron doped SmFeAsO compounds through the partial fluorine doping for oxygen $[4,5]$. In contrast to cuprate superconductor, superconductivity can also be induced by the substitution of Fe with other transition metal including Rh [6], Co [7] and $\mathrm{Ni}$ [8]. Apart from the chemical or internal pressure, the effect of high pressure on superconductivity of iron based superconductors has also been studied and revealed the doping dependence of pressure effect [9]. However, isovalent doping like $\mathrm{P}$ on As site, which is generally believed not to introduce charge carriers, has been proved to be effective to induce superconductivity in both RE1111 [10] and $\mathrm{BaFe}_{2} \mathrm{As}_{2}$ (122) systems [11]. Since $\mathrm{P}^{3-}$ ion is smaller than $\mathrm{As}^{3-}$ ion, and thus introduces internal strain or distortion, i.e., chemical pressure. Li et al has studied the effect of $\mathrm{P}$ doping in $\mathrm{SmFeAsO}$ and showed the maximum onset $T_{\mathrm{c}}$ of $4.1 \mathrm{~K}$ at $56 \%$ of $\mathrm{P}$ doping [12], however no work has been reported on the effect of $\mathrm{P}$ substitution in $\operatorname{SmFeAs}(\mathrm{O}, \mathrm{F})$ which has $T_{\mathrm{c}}$ exceeding $50 \mathrm{~K}$ [5]. Actually the second element doping could modify the crystal structure/electronic structure of the host material giving rise to any/all superconducting properties as reported in $\mathrm{Y}$ doped $\mathrm{CeFeAs}(\mathrm{O}, \mathrm{F})$ [13] and $\mathrm{Gd}$ doped $\operatorname{CeFeAs}(\mathrm{O}, \mathrm{F})[14]$. 
To understand the chemical doping effect in superconducting layer FeAs, we have studied the interplay between electron doping by fluorine $(\mathrm{F})$ and the effect of internal chemical pressure by phosphorus $(\mathrm{P})$ substitution in $\mathrm{SmFeAsO}_{0.88} \mathrm{~F}_{0.12}$. These results are compared with doping effects of Mn and Ni in FeAs layers of F-doped Sm1111 reported in our paper [8]. Our results show that the superconducting properties decrease rapidly from chemical doping in FeAs layers which might be due to disorder introduced by partial substitutions of $\mathrm{P}$ for As or $\mathrm{Mn}, \mathrm{Ni}$ for Fe in the conducting layer.

\section{Experimental}

Polycrystalline samples with nominal compositions of $\mathrm{SmFeAs}_{1-x} \mathrm{P}_{x} \mathrm{O}_{0.88} \mathrm{~F}_{0.12}(x=0,0.05$, $0.1,0.15,0.2,0.25$ and 0.3$)$ were synthesized by single step solid state reaction $[5,8]$ starting from $\mathrm{SmAs}, \mathrm{Fe}_{2} \mathrm{O}_{3}, \mathrm{FeF}_{2} \mathrm{Fe}$, and $\mathrm{FeP}$. The samples with different doping concentrations were mixed and pressed into pellets under the same pressure of $40 \mathrm{MPa}$, wrapped in Ta foils and heated under exactly the same conditions to eliminate the possible errors in the sample-making process. The samples were sintered at $900^{\circ} \mathrm{C}$ for $45 \mathrm{~h}$ in evacuated quartz ampoules. The resulting samples were characterized by powder X-ray diffraction (XRD) with $\mathrm{Cu}-K_{\alpha}$ radiation. The XRD intensity data were collected in the $2 \theta$ range of $10-80^{\circ}$ at a step of $0.02^{\circ}$ using $\mathrm{Cu} \mathrm{K} \alpha$ radiation. Silicon powder was used as an internal standard. The $d$-values were calculated from angle of peaks. The lattice parameters were obtained by a least squares fit of the observed $d$ values.

The temperature dependence of resistivity $(\rho)$ and Hall coefficient $\left(R_{\mathrm{H}}\right)$ up to $9 \mathrm{~T}$ was measured by Quantum Design PPMS Model 6000. Magnetic measurements were performed by a SQUID magnetometer (Quantum Design: MPMS-XL5s). The zero-field-cooled (ZFC) and field- 
cooled (FC) magnetization was measured under 1 Oe in a temperature range of $2-60 \mathrm{~K}$. The thermoelectric power measurement of each sample was carried out by the steady-state technique where a temperature gradient was created across the rectangular shaped samples with dimensions of $4 \times 3 \times 0.7 \mathrm{~mm}^{3}$, in the temperature range of $5-300 \mathrm{~K}$ using a homemade setup attached to a cryostat which is immersed in the cryogenic liquid. The samples were directly attached to copper- constantan (Type T) thermocouple using silver paste. The difference of voltage, $\Delta V$, between the hot and cold end of the sample was measured by a Keithley 2182 Nanovoltmeter using copper wires.

\section{Results and discussions}

The X-ray diffraction (XRD) patterns of all $\mathrm{SmFeAs}_{1-x} \mathrm{P}_{x} \mathrm{O}_{0.88} \mathrm{~F}_{0.12}$ samples are shown in Fig. 1a. The peaks are indexed on the basis of the tetragonal $\mathrm{ZrCuSiAs-type} \mathrm{structure} \mathrm{with} \mathrm{the} \mathrm{space}$ group $P 4 / \mathrm{nmm}$. The expected $\mathrm{SmFeAsO}$ was formed as the main phase for all the samples. The impurity phases of $\mathrm{SmAs}, \mathrm{SmOF}$ and $\mathrm{Fe}_{2} \mathrm{P}$ were also observed in the samples with high $\mathrm{P}$ contents. The variation of lattice constants $(a$ and $c)$ and the cell volume $(V)$ with doping of $\mathrm{P}$ are shown in Fig. 1b, which indicates that the $\mathrm{P}$ atoms were successfully introduced into the lattice.

The lattice constants at room temperature were evaluated as $a=3.930(8) \AA$ and $c=8.478(8) \AA$ for F-doped $\mathrm{SmFeAsO}_{0.88} \mathrm{~F}_{0.12}$. The $a$-axis length is almost constant whereas the $c$-axis length largely shrinks with an increase in P doping level. This shrinkage causes a reduction in the cell volume $(V)$ which induces an additional chemical pressure into the system. Due to large shrinkage of $c$-axis, this chemical pressure could be anisotropic due to isovalent $\mathrm{P}$ substitution at As sites. 
Temperature dependence of resistivity $(\rho)$ of $\operatorname{SmFeAs}_{1-x} \mathrm{P}_{x} \mathrm{O}_{0.88} \mathrm{~F}_{0.12}$ is shown in Fig. 2. One can see that the resistivity for only F-doped $\mathrm{SmFeAsO}(x=0)$ decreases approximately linearly from room temperature to transition temperature and superconductivity appears at $\sim 51 \mathrm{~K}$. The $\rho$ value for $x=0.05$ decreased in the whole temperature region compared to that of $x=0$. As $\mathrm{P}$ content increased further, $\rho$ enhanced and $T_{\mathrm{c}}$ shifted very rapidly to lower temperature down to $20 \mathrm{~K}$ for $x=0.2$, as shown in the inset of Fig. 2. Meanwhile, superconductivity disappears at $x \geq 0.25$ and an anomaly is observed around $140 \mathrm{~K}$ which might be due to structural phase transition followed by antiferromagnetic ordering similar to that of the parent compound SmFeAsO $[5,15]$. The observed onset $T_{\mathrm{c}}$ decreases rapidly even as the reduction of the cell volume $(V)$ as shown in Fig. 1b. Therefore, the $T_{\mathrm{c}}$ reduction mechanism involved in this system is opposite to the other oxypnictide superconductors i.e. lanthanide contraction due to the substitution of relatively smaller ions $[13,16-17]$. On the other hand, a sample with high $\mathrm{P}$ content $x=0.3$ shows a strong upturn in its resistivity curve at low temperature region similar to $\mathrm{Mn}$ doped $\mathrm{SmFeAsO}_{0.88} \mathrm{~F}_{0.12}$ [8], which would result from Kondo effect or charge carrier localization due to the disorder in the conducting layers as P doped on the As sites. P doping could also increase the intrinsic itinerancy of the $d$ electrons and significantly enhances the interlayer d-f coupling or Kondo effect $[18,19]$. Since only F-doping introduces extra electrons into FeAs layer and moderately increases the interlayer d-f coupling. This means that Kondo coupling is weaker in F-doping case. As P is doped in F-doped Sm1111, Kondo effect is more evident (Fig. 2) similar to the reported $\mathrm{P}$ doped $\operatorname{CeFeAs}(\mathrm{O}, \mathrm{F})[18,19]$ and, thus superconductivity is suppressed and the enhancement of resistivity occurs. P-doping induced the high resistivity similar to the case of $\mathrm{P}$ doped $\mathrm{CeFeAs}(\mathrm{O}, \mathrm{F})[18,19]$, presumably corresponding to larger structure changes in the FeAs layer. 
The bulk superconductivity in these samples is also confirmed by the temperature dependence of the magnetic susceptibility $(\chi)$ measurements. ZFC and FC magnetization curves measured under 1 Oe are shown in Fig. 3. All samples showed very sharp transitions in ZFC curves. Large $\chi$ values observed at low temperatures are the strong evidence of their bulk superconductivity. Note that the demagnetization effects of each sample contributed to larger magnetization expected from the perfect diamagnetism, $\chi=-1$. The suppression of $T_{\mathrm{c}}$ is remarkable (as shown in Fig. 4a) similar to Ni or Mn doped Sm1111 [8] and superconductivity is not observed for $x \geq 0.25$ which is consist with the resistivity data. These findings seem almost similar behaviors for all three dopants $\mathrm{P}, \mathrm{Mn}$ and $\mathrm{Ni}$ in the conducting FeAs layer. The variation of resistivity at 60 $\mathrm{K}\left(\rho_{60 \mathrm{~K}}\right)$ with Ni, Mn and P contents is plotted in Fig. 4b. Samples with Ni doping showed the metallic nature whereas the resistivity monotonically enhanced for $\mathrm{Mn}$ and $\mathrm{P}$ doping. It suggest that isovalent $(\mathrm{P})$ and hole $(\mathrm{Mn})$ doping in FeAs layers behave like scattering centers and interrupt the electron conduction.

The temperature dependence of the resistivity under magnetic fields up to $9 \mathrm{~T}$ was measured for a series of samples. The resistivity curves for a $\operatorname{SmFeAs}{ }_{0.95} \mathrm{P}_{0.05} \mathrm{O}_{0.88} \mathrm{~F}_{0.12}$ sample are shown in Fig. 5. The resistivity starts to drop at $47 \mathrm{~K}$ and then becomes zero at $44 \mathrm{~K}$ in zero magnetic field. The onset transition temperature gradually decreases with magnetic field, while the zero resistance temperature reduced significantly mainly due to weak coupling between grains rather than thermally activated flux motions in the crystal. The field dependence of offset $T_{\mathrm{c}}$ give information about the weak links between grains as well as vortex flow behavior, while the onset $T_{\mathrm{c}}$ with the applied magnetic field is controlled by the upper critical field of the individual grains. The upper critical field $\left(H_{\mathrm{c} 2}\right)$ and irreversibility field $\left(H_{\text {irr }}\right)$ correspond to the temperatures, where the resistivity drops to $90 \%$ and $10 \%$ of normal state resistivity $\rho_{\mathrm{n}}(T, H)$ at the onset $T_{\mathrm{c}}$ in applied 
magnetic fields, respectively. The $H-T$ diagram for different $\mathrm{P}$ doped samples is shown in the inset of Fig. 5. The slope of upper critical field $\left(\mu_{0} \mathrm{~d} H_{\mathrm{c} 2} / \mathrm{d} T\right)$ decreases with respect to $\mathrm{P}$ composition, which are $-8.4,-6.0,-4.5,-2.7,-2.6 \mathrm{~T} \mathrm{~K}^{-1}$ in $x=0,0.05,0.1,0.15$ and 0.2 , respectively. The upper critical field at $0 \mathrm{~K}\left(\mu_{0} H_{\mathrm{c} 2}(0)\right)$ is estimated using the conventional oneband Werthamer-Helfand-Hohenberg $(\mathrm{WHH})$ equation i.e. $H_{\mathrm{c} 2}(0)=-0.693 T_{\mathrm{c}}\left(\mathrm{d} H_{\mathrm{c} 2} / \mathrm{d} T\right)_{T=T_{\mathrm{c}}}[20]$. Using the values of $T_{\mathrm{c}}, H_{\mathrm{c} 2}(0)$ of the samples with $x=0,0.05,0.1,0.15$ and 0.2 are estimated to be $292 \mathrm{~T}, 194 \mathrm{~T}, 116 \mathrm{~T}, 36 \mathrm{~T}$ and $31 \mathrm{~T}$, respectively. The $H_{\mathrm{c} 2}$ value decreases rapidly with the increasing concentration of $\mathrm{P}$ as reported for $\mathrm{Mn}$ and Ni doping in the FeAs layers [8]. Using the value of $H_{\mathrm{c} 2}(0)$, we can also calculate the mean field Ginberg-Landau coherence length $\xi_{\mathrm{ab}}=$ $\left(\phi_{0} / 2 \pi H_{\mathrm{c} 2}\right)^{1 / 2}$. Using $\phi_{0}=2.07 \times 10^{-7} \mathrm{G} \mathrm{cm}^{2}$ and the $H_{\mathrm{c} 2}$ value, we obtain a coherence length of $10.6 \AA$ for a sample with $x=0$. This value increases with respect to P doping in the FeAs layer due to the suppression of $H_{\mathrm{c} 2}$.

To confirm the dominant conduction mechanism, we have investigated the temperature dependence of Hall coefficient $\left(R_{\mathrm{H}}\right)$ and thermoelectric power $(S)$ for $\mathrm{SmFeAsO}_{0.88} \mathrm{~F}_{0.12}$ and $\mathrm{SmFeAs}_{1-x} \mathrm{P}_{x} \mathrm{O}_{0.88} \mathrm{~F}_{0.12}(x=0.05,0.1$ and 0.15$)$ as shown in Figs. $6 \mathrm{a}$ and $\mathrm{b}$, respectively. We have studied the Hall resistivity $\left(\rho_{x y}\right)$ of superconducting $\operatorname{SmFeAs}_{1-x} \mathrm{P}_{x} \mathrm{O}_{0.88} \mathrm{~F}_{0.12}$ samples at different temperature from $50-300 \mathrm{~K}$, as shown in the inset of Fig. $6 \mathrm{a}$ for $x=0.1$. It is clear that $\rho_{x y}$ is linear with the magnetic field and is observed for the measured temperature range, indicating that the normal-state conduction of these samples is dominated by electron-like charge carriers. From this set of data, the Hall coefficient $R_{\mathrm{H}}=\rho_{\mathrm{xy}} / H$ was determined as shown in Fig. 6a. The $R_{\mathrm{H}}$ is nearly a constant with varying temperature for a normal metal with Fermi-liquid feature. This situation changes for a multiband material or in non-Fermi-liquid behaviour, such as cuprate superconductors [21]. The temperature dependence of Hall coefficient suggests either a 
multiband effect or some unusual scattering process. Using the single band equation $n=\left|1 / R_{\mathrm{H}} e\right|$ to evaluate the charge-carrier density, we obtain $n$ values at $60 \mathrm{~K}$ which are $7.8 \times 10^{22} \mathrm{~cm}^{-3}, 5.8 \times$ $10^{22} \mathrm{~cm}^{-3}$ and $4.8 \times 10^{22} \mathrm{~cm}^{-3}$ for $x=0,0.05$ and 0.1 , respectively. It is clear that the charge carrier density at high temperature is almost unchanged by $\mathrm{P}$ doping above $150 \mathrm{~K}$ due to $\mathrm{P}$ doping but at the below $150 \mathrm{~K}, n$ reduced with respect to doping. This might be a reason for the negative effect of P-doping changing the symmetry of FeAs tetrahedral structure. On the hand, the $R_{\mathrm{H}}$ is negative in the entire temperature range, suggesting that the superconductivity in all these samples is dominated by negative charge carriers.

The temperature dependence of thermoelectric power $(S)$ is shown in Fig. $6 \mathrm{~b}$ for samples with various P doping levels. For $x=0$, the absolute value of thermoelectric power $(|S|)$ increases with decreasing temperature, while it develops a broad maximum below $100 \mathrm{~K}$. After that $|S|$ decreases rapidly and becomes zero around $T_{\mathrm{c}}$. The onset $T_{\mathrm{c}}$ values observed in resistivity and thermoelectric power measurements are almost identical. When $\mathrm{P}$ is doped at As site, the profile of $S(T)$ curves behave like $\mathrm{SmFeAsO}_{0.88} \mathrm{~F}_{0.12}$ and $|S|$ value is approximately same as $x=0$. We also note that the minima of the negative thermoelectric power does not shift to lower temperature as compared to $x=0$. Since P does not introduce charge carriers and avoids possible scattering effects by charged impurities in the FeAs planes. These might be a reason that $|S|$ has not very effective with respect to the $\mathrm{P}$ concentration which is different result compared with that of $\mathrm{Mn}$ or Ni doping [8]. The thermoelectric power is negative for all temperatures above $T_{\mathrm{c}}$, suggesting the electron type charge carriers are playing a major role and support the Hall coefficient studies.

A central feature of iron based superconductors is their multiband character with the electron and hole Fermi surface pockets. The Ni doping makes the hole Fermi surface shrink and the 
electron Fermi surface expand whereas the hole Fermi surface expands with Mn doping and the electron Fermi surface shrink rapidly [8]. With P doping, the numbers of electron and hole Fermi surface pockets should not change and thus Fermi surface topology should not significantly change over the entire range of $\mathrm{P}$ content. However, the results for the isovalent $(\mathrm{P})$ doping suggest an additional evidence for the electron-hole asymmetry. On the other hand, the local structure of $\mathrm{FeAs}_{4}$ tetrahedron also plays an important role in the superconductivity for oxypnictides. Zhao et al. [22] found that $T_{\mathrm{c}}$ increases as As-Fe-As bond angle approaches very close to the standard value of $109.47^{\circ}$, as expected for a perfect tetrahedron. Superconductivity with a critical temperature $T_{\mathrm{c}}$ of up to $58 \mathrm{~K}$ emerges, when the bond angle is reached closed to the ideal band value by electron doping of the FeAs layer via fluoride ion $\left(\mathrm{F}^{-}\right)$substitution of oxygen sites [5]. On the basis of above discussion, $\mathrm{P}$ doping in $\mathrm{SmFeAsO}_{0.88} \mathrm{~F}_{0.12}$ induces, possibly, the anisotropic chemical pressure with strong suppression of superconductivity. These results suggest that the isovalent doping changes the symmetry of FeAs layer and expand the bond angle of As-Fe-As away from the ideal value similar to the case Mn and Ni doping. These structure changes could also effect on the Fermi surface pockets and some of the hole Fermi surface pockets become more warped as $x$ increases over the entire range of $\mathrm{P}$ at As site as proposed for $\mathrm{P}$ doped Ba122 system [23]. Hence, the considerable decrease in $T_{\mathrm{c}}$ induced by $\mathrm{Mn}$, $\mathrm{Ni}$ and $\mathrm{P}$ doping in superconducting (FeAs) layer could be ascribed to the disorder in the Fe sublattice similar to the case of Ru doped $\operatorname{SmFeAs}(\mathrm{O}, \mathrm{F})$ [24] because the superconducting properties would be strongly affected by the disorder [25]. 


\section{Conclusions}

The fluorine (F) doping in the $\mathrm{SmO}$ layer of $\mathrm{SmFeAsO}$ leads to superconductivity up to $58 \mathrm{~K}$ due to the enhancement of chemical pressure. Here, the effect of chemical pressure on $\mathrm{SmFeAsO}_{0.88} \mathrm{~F}_{0.12}$ induced by $\mathrm{P}$ doping on the As site is studied and, strongly suppressed the $T_{\mathrm{c}}$ and $H_{\mathrm{c} 2}$ similar to $\mathrm{Mn}$ or $\mathrm{Ni}$ doping. However, the in-plane lattice parameter is almost unaffected whereas the out-of-plane lattice parameter systematically decreases with $\mathrm{P}$ doping. Hall coefficient measurements showed the slight change of negative charge carriers in the low temperature region which might be due to change of symmetry of FeAs layers. The observed thermoelectric power also indicates dominance of electron charge carriers along with strong electron correlation in this compound. Summarily, our results suggest that disorder in the Fesublattice plays an serious role in suppression of superconductivity in Sm1111 and hence, electron or hole doping without disrupting the conducting FeAs layer is considered to be a better way of realizing superconductivity with high $T_{\mathrm{c}}$ in iron pnictides.

\section{Acknowledgements}

SJS is supported by Japan Society for the Promotion of Science (JSPS), Government of Japan. This work was partially supported by Japan Science and Technology Agency (JST), PRESTO, SICORP and TRIP. 


\section{References}

[1] Y. Kamihara, T. Watanabe, M. Hirano and H. Hosono, J. Am. Chem. Soc. 130 (2008) 3296.

[2] G. R. Stewart, Rev. Mod. Phys. 83 (2011) 1589.

[3] H. Takahashi, K. Igawa, K. Arii, Y. Kamihara, M. Hirano and H. Hosono, Nature 453 (2008) 376.

[4] Z. A. Ren, W. Lui, J. Yang, W. Yi, X. L. Shen, Z. C. Li, G. C. Che, X. L. Dong, L. L. Sun, F. Zhou and Z. X. Zhao, Chin. Phys. Lett. 25 (2008) 2215.

[5] S. J. Singh, J. Shimoyama, A. Yamamoto, H. Ogino and K. Kishio, IEEE Trans. Appl. Supercond. 23 (2013) 7300605.

[6] M. Tropeano, M. R. Cimberle, C. Ferdeghini, G. Lamura, A. Martinelli, A. Palenzona, I. Pallecchi, A. Sala, I. Sheikin, F. Bernardini, M. Monni, S. Massidda and M. Putti, Phys. Rev. B 81 (2010) 184504.

[7] V. P. S. Awana, A. Pal, A. Vajpayee, R. S. Meena, H. Kishan, M. Husain, R. Zeng, S. Yu, K. Yamaura and E. Takayama-Muromachi, J. Appl. Phys. 107 (2010) 09E146.

[8] S. J. Singh, J. Shimoyama, A. Yamamoto, H. Ogino and K. Kishio, Physica C 494 (2013) 57.

[9] C. W. Chu and B. Lorenz, Physica C 469 (2009) 385.

[10] C. Wang, S. Jiang, Q Tao, Z. Ren, Y. Li, L. Li, C. Feng, J. Dai, G. Cao and Z. Xu, Europhys. Lett. 86 (2009) 47002.

[11] S. Kasahara, T. Shibauchi, K. Hashimoto, K. Ikada, S. Tonegawa, R. Okazaki, H. Shishido, H. Ikeda, H. Takeya, K. Hirata, T. Terashima and Y. Matsuda, Phys. Rev. B 81 (2010) 184519.

[12] Y. Li, X. Lin, T. Zhou, J. Shen, Y. Luo, Q. Tao, G. Cao and Z. Xu, Physica C 470 (2010) S493. 
[13] J. Prakash, S. J. Singh, A. Banerjee, S. Patnaik and A. K. Ganguli, Appl. Phys. Lett. 95 (2009) 262507.

[14] J. L. Yang, W. J. Ren, D. Li, Z. D. Zhang, J. Appl. Phys. 109 (2011) 07E154.

[15] B. Lorenz, K. Sasmal, R. P. Chaudhury, X. H. Chen, R. H. Liu, T. Wu and C. W. Chu, Phys. Rev. B 78 (2008) 012505.

[16] M. Tropeano, C. Fanciulli, F. Canepa, M. R. Cimberle, C. Ferdeghini, G. Lamura, A. Martinelli, M. Putti, M. Vignolo, A. Palenzona, Phys. Rev. B 79 (2009) 174523.

[17] P. M. Shirage, K. Miyazawa, H. Kito, H. Eisaki, A. Iyo, Phys. Rev. B 78 (2008) 172503.

[18] Y. Luo, X. Lin, H. Han, S. Jiang, Y. Li, J. Dai, G. Cao, Z. Xu, J. Phys. Chem. Solids 172 (2011) 430 .

[19] Y. Luo, H. Han, S. Jiang, X. Lin, Y. Li, J. Dai, G. Cao and Z. Xu, Phys. Rev. B 83 (2011) 054501.

[20] N. R. Werthamer, E. Helfand, P. C. Hohenberg, Phys. Rev. 147 (1966) 295.

[21] N. P. Ong, Physical properties of High Temperature Superconductors, in: D. M. Ginsberg (Ed.), World Scientific, Singapore, 1990, p. 459.

[22] J. Zhao, Q. Huang, C. D. Cruz, S. Li, J. W. Lynn, Y. Chen, M. A. Green, G. F. Chen, G. Li, Z. Li, J. L. Luo, N. L. Wang and P. Dai, Nature 7 (2008) 953.

[23] Z. R. Ye, Y. Zhang, F. Chen, M. Xu, Q. Q. Ge, J. Jiang, B. P. Xie and D. L. Feng, Phys. Rev. B 86 (2012) 035136.

[24] D. Daghero, M. Tortello, G. A. Ummarino, V. A. Stepanov, F. Bernardini, M. Tropeano, M. Putti, R. S. Gonnelli, Supercond. Sci. Technol. 25 (2012) 084012.

[25] Y. Mizuguchi, Y. Hara, K. Deguchi, S, Tsuda, T. Yamaguchi, K. Takeda, H. Kotegawa, H. Tou and Y. Takano, Supercond. Sci. Technol. 23 (2010) 054013. 


\section{Figure Captions:}

Fig. 1. (a) Powder XRD patterns for $\mathrm{P}$ doped $\mathrm{SmFeAs}_{1-x} \mathrm{P}_{x} \mathrm{O}_{0.88} \mathrm{~F}_{0.12}(x=0,0.05,0.1,0.15,0.2$, 0.25 and 0.3$)$; (b) the variation of lattice constants ( $a$ and $c$ ) and the unit cell volume $(V)$ with $\mathrm{P}$ content $(x)$.

Fig. 2. The variation of resistivity $(\rho)$ with temperature up to $300 \mathrm{~K}$ for $\operatorname{SmFeAs}_{1-x} \mathrm{P}_{x} \mathrm{O}_{0.88} \mathrm{~F}_{0.12}(x$ $=0,0.05,0.1,0.2$ and 0.3$)$. The inset shows the temperature dependence of $\rho$ in low temperature region except for $x=0.3$.

Fig. 3. ZFC and FC magnetic susceptibility $(\chi)$ curves under $H=1$ Oe for $\operatorname{SmFeAs}_{1-x} \mathrm{P}_{x} \mathrm{O}_{0.88} \mathrm{~F}_{0.12}$.

Fig. 4. (a) The variation of $T_{\mathrm{c}}$ and (b) the resistivity $\left(\rho_{60 \mathrm{~K}}\right)$ at $60 \mathrm{~K}$ with various $\mathrm{P}$ (triangle symbol), Mn (rectangular symbol) and Ni (circle symbol) doping levels.

Fig. 5. The variation of resistivity with respect to temperature in the presence of applied magnetic field up to $9 \mathrm{~T}$ for $\mathrm{SmFeAs}_{0.95} \mathrm{P}_{0.05} \mathrm{O}_{0.88} \mathrm{~F}_{0.12}$. The inset shows the upper critical field $\left(H_{\mathrm{c} 2}\right)$ (closed symbol) and irreversibility field $\left(H_{\mathrm{irr}}\right)$ (open symbol) for the samples $\mathrm{SmFeAs}_{1-}$ ${ }_{x} \mathrm{P}_{x} \mathrm{O}_{0.88} \mathrm{~F}_{0.12}$ determined by $90 \%$ and $10 \%$ of the normal state resistivity.

Fig. 6. (a) Hall coefficient as a function of temperature for $\operatorname{SmFeAs}_{1-x} \mathrm{P}_{x} \mathrm{O}_{0.88} \mathrm{~F}_{0.12}(x=0,0.05$, 0.1) samples. The inset depicts Hall resistivity as a function of field for $x=0.1$ at different temperature (b) the variation of thermoelectric power $(S)$ with respect to temperature for $\mathrm{SmFeAs}_{1-x} \mathrm{P}_{x} \mathrm{O}_{0.88} \mathrm{~F}_{0.12}$ sample. 
Fig. 1.
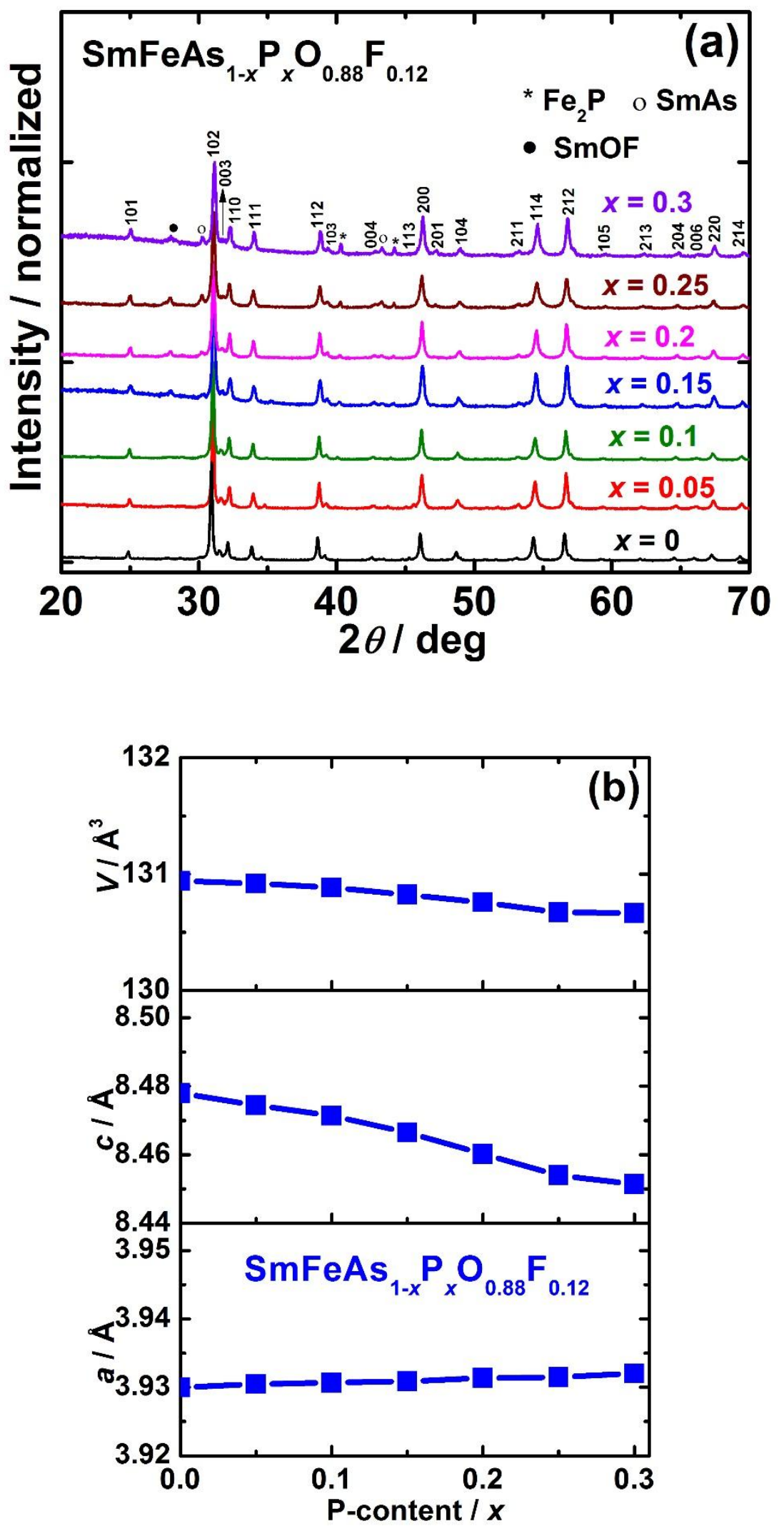
Fig. 2.

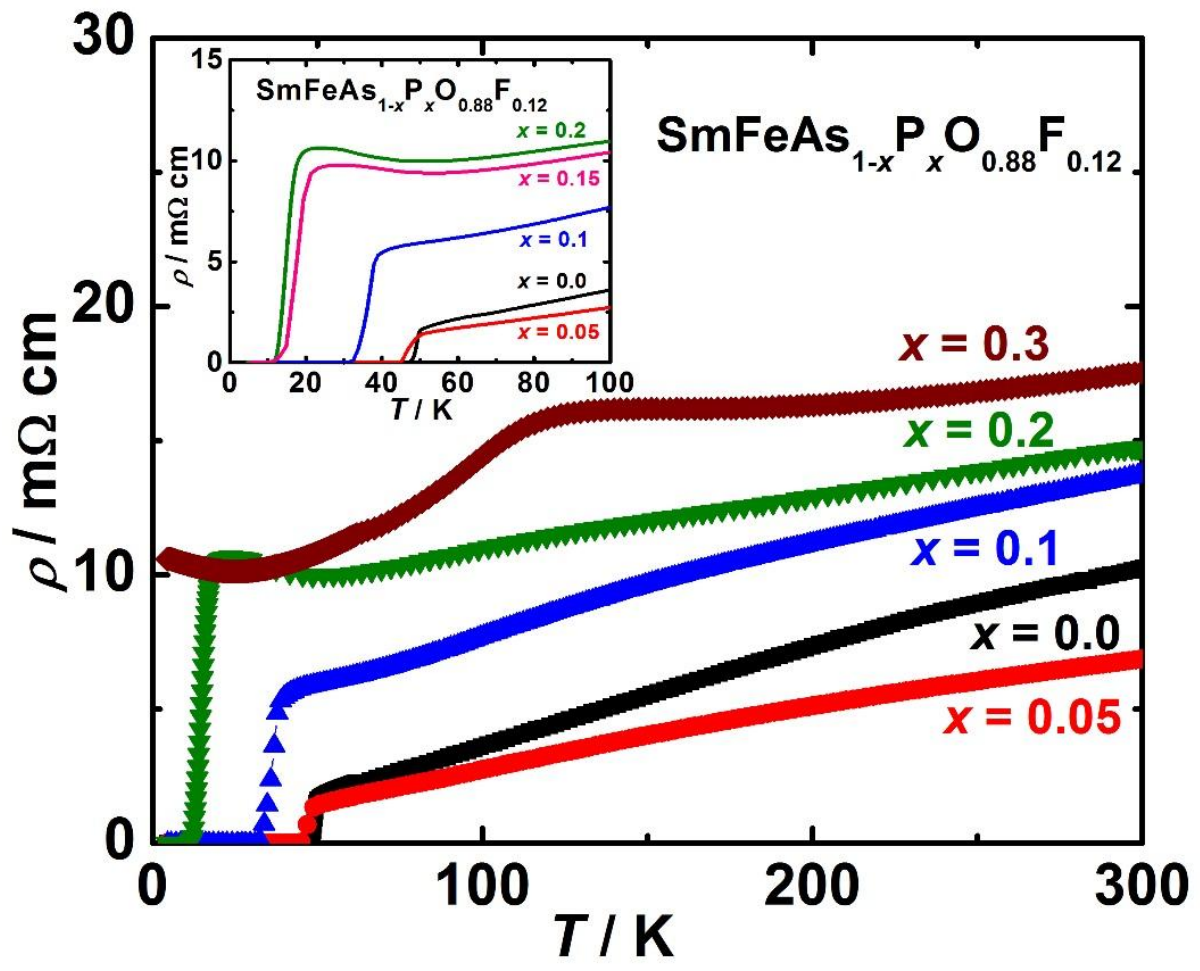


Fig. 3.

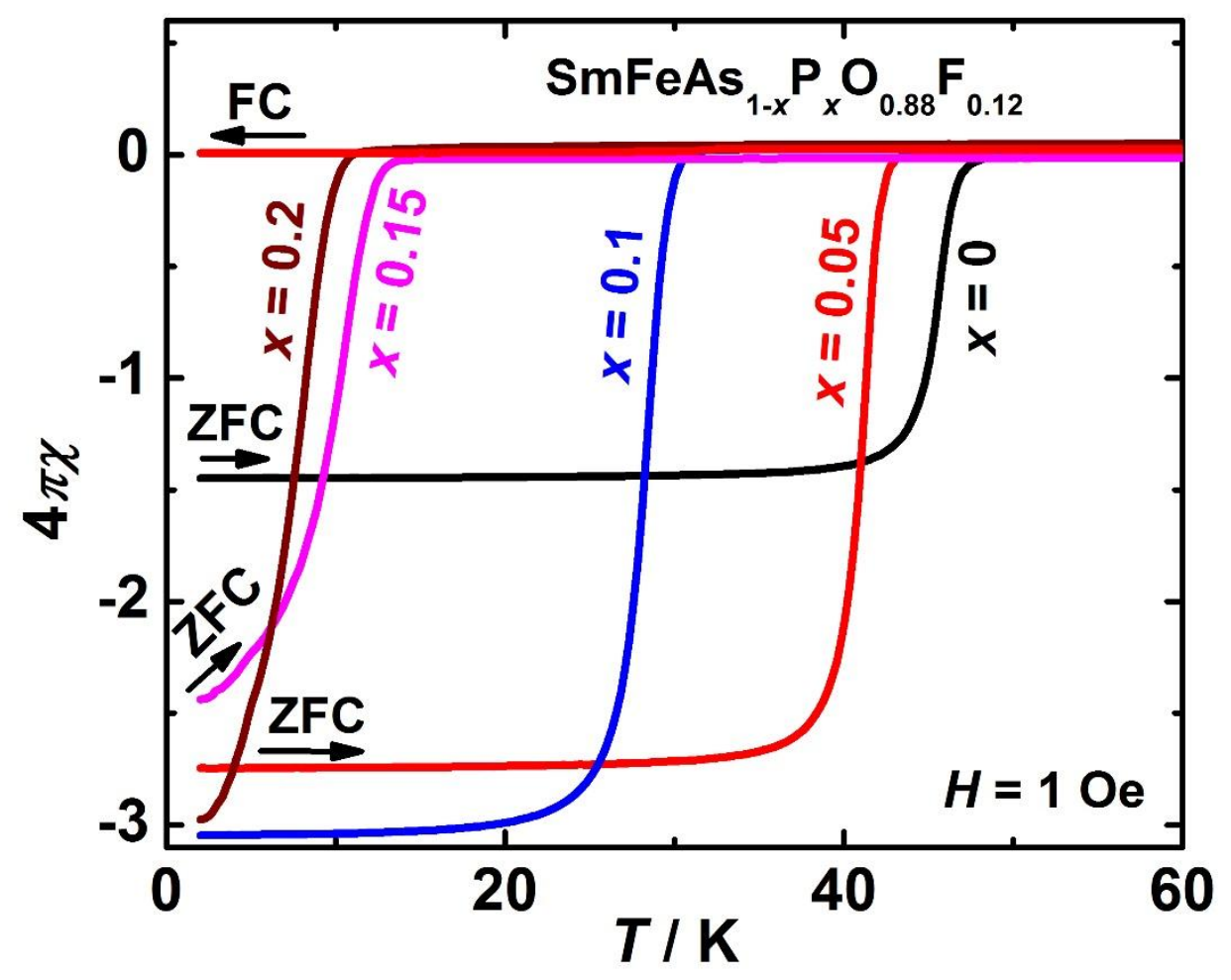


Fig. 4.
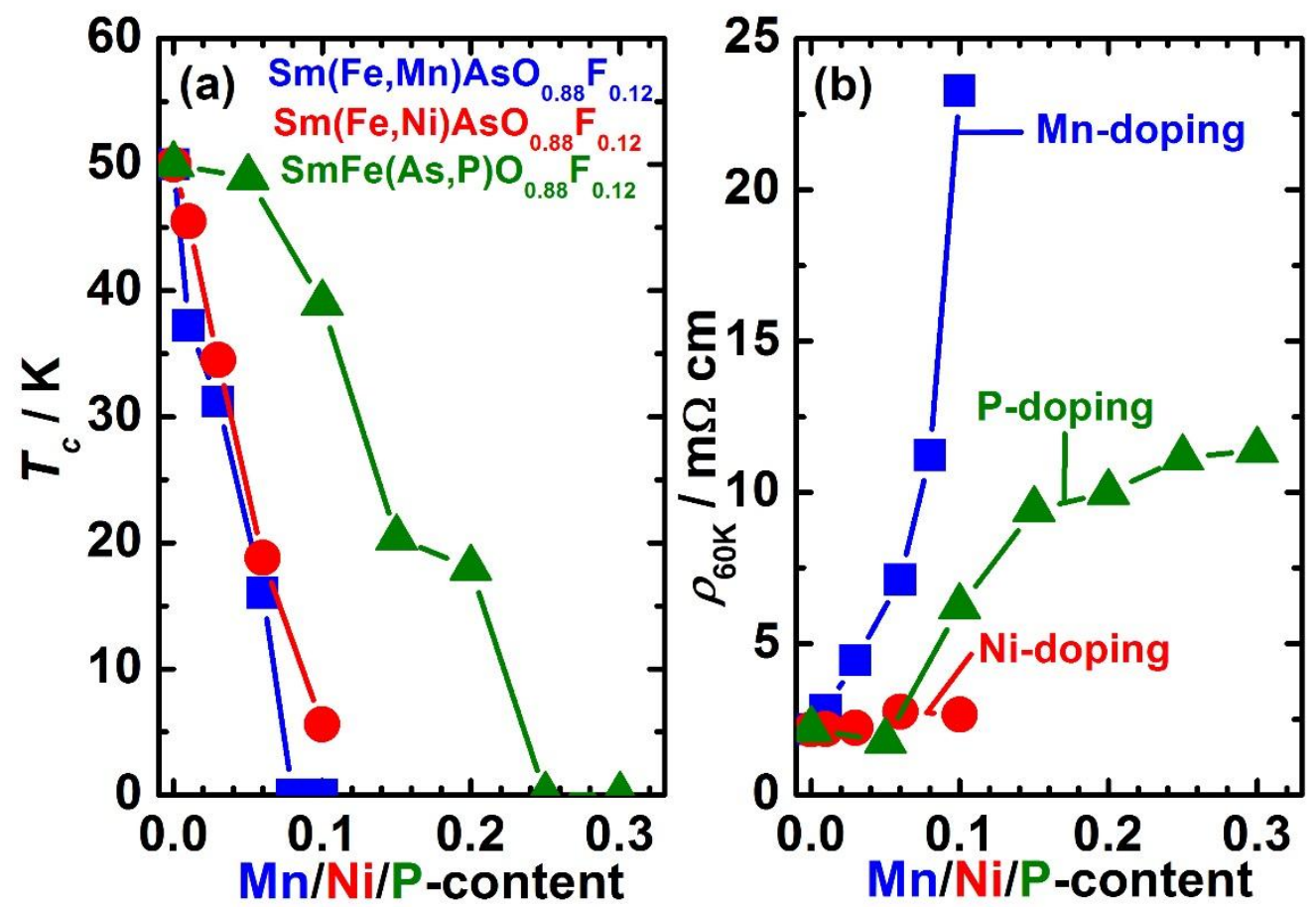
Fig. 5.

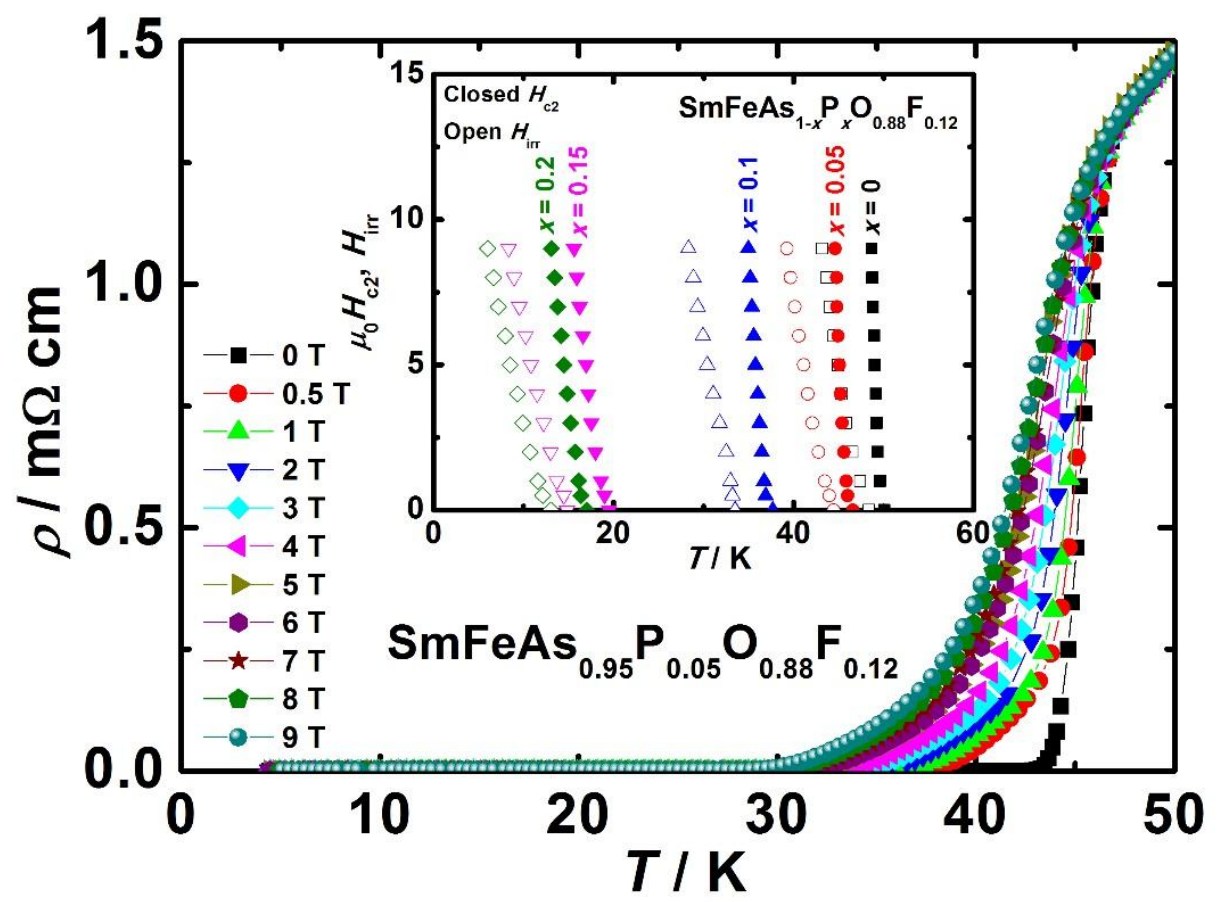


Fig. 6.
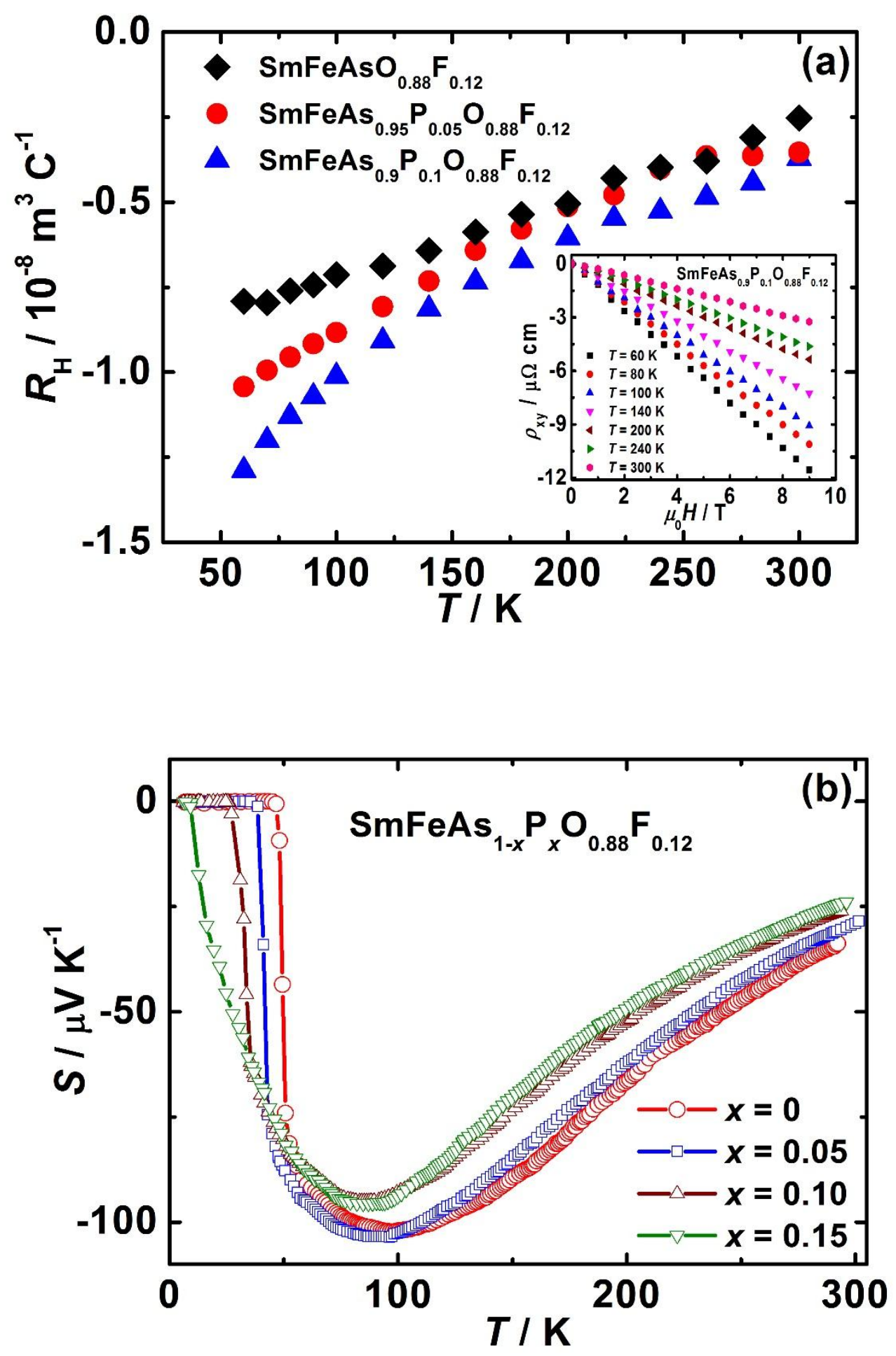